\title{
Combinatorial Sec pathway analysis for improved heterologous protein secretion in Bacillus subtilis: identification of bottlenecks by systematic gene overexpression
}

\author{
Jingqi Chen ${ }^{1,2,3}$, Gang Fu' ${ }^{2,4}$, Yuanming Gai ${ }^{2,4}$, Ping Zheng ${ }^{2,3}$, Dawei Zhang ${ }^{2,3,4^{*}}$ and Jianping Wen ${ }^{1}$
}

\begin{abstract}
Background: Secretory expression of valuable proteins by B. subtilis and its related species has attracted intensive work over the past three decades. Although very high yields can be achieved with homologous proteins, production of heterologous proteins by B. subtilis is unfortunately not the straight forward. The Sec pathway is the major route for protein secretion in B. subtilis. Therefore, the aim of this work was to identify the bottlenecks of the Sec pathway and improve the secretion of heterologous proteins by molecular genetic techniques.

Results: Two $\alpha$-amylases (AmyL and AmyS) both under the control of the $\mathrm{P}_{\text {Hpall }}$ promoter and equipped with their native signal peptides $\mathrm{SP}_{\text {amyl }}$ and $\mathrm{SP}$ amys were successfully secreted with significantly different expression levels. To improve the secretion efficiency, 23 main genes or gene operons involved in or closely related to the Sec pathway were overexpressed singly by increasing an additional copy on the chromosome, and the overexpression of prs $A$ enhanced the production of $\alpha$-amylases (AmyL and AmyS) by 3.2- and 5.5-fold, respectively. With the induction by xylose of different concentrations, prsA overexpression level was optimized and the secretion efficiency of $\alpha$-amylase was further improved. Moreover, combinatorial overexpression of prsA and nine screened genes or gene operons, respectively, was performed, and the overexpression of prsA combined with partial dnaK operon improved the $\alpha$-amylase activity of AmyL and AmyS by 160 and 173\%, respectively, compared with the overexpression of prsA singly. Finally, the performance of the recombinant B. subtilis 1A237 was evaluated with the fed-batch fermentation in $7.5 \mathrm{~L}$ fermentor, and the level of secreted AmyL and AmyS reached 1,352 and 2,300 U/mL with the productivity of 16.1 $\mathrm{U} / \mathrm{mL} h$ and $27.4 \mathrm{U} / \mathrm{mL} h$, respectively.
\end{abstract}

Conclusions: Our systematic gene overexpression approach was designed to investigate the bottleneck of Sec pathway in B. subtilis. The deficiency of PrsA lipoprotein and chaperones of DnaK series was main rate-limiting factors for heterologous proteins secretion. Systematic and deep insight into how components of Sec pathway interact with each other may be the key to improving the yield of heterologous proteins thoroughly.

Keywords: Bacillus subtilis, Bottleneck, Heterologous protein, Overexpression, Sec pathway

\section{Backgroud}

The Gram-positive bacterium Bacillus subtilis is widely known for its capacity to produce and secrete large amounts of industrially relevant proteins, mostly

\footnotetext{
*Correspondence: zhang_dw@tib.cas.cn

${ }^{2}$ Tianjin Institute of Industrial Biotechnology, Chinese Academy

of Sciences, Tianjin 300308, People's Republic of China

Full list of author information is available at the end of the article
}

endogenous enzymes like amylases, proteases and lipases. In particular, the ability of B. subtilis to secrete proteinaceous products into the growth medium greatly facilitates downstream processing. The use of B. subtilis also has many other advantages, such as its GRAS (generally regarded as safe) status [1, 2], high amenability for genetic engineering [3], no pronounced codon bias [4] and the easy and inexpensive culturing methods 
and large-scale fermentation. Thus, scientists pay more attention to this organism with the aim to the commercial exploitation of B. subtilis as major "cell factories" for secreted heterologous proteins of interest.

In $B$. subtilis, the major route of protein transport across the cytoplasmic membrane is the general secretion (Sec) pathway [5, 6]. This involves four types of secretory machinery components: (1) Signal recognition particle (SRP). SRP, a highly conserved and essential ribonucleoprotein complex, is in charge of recognizing the SP sequence of a nascent chain and targeting it to the membrane. B. subtilis SRP consists of a small cytoplasmic RNA (scRNA) [7], a GTPase Ffh [8] and two molecules of Hbsu protein [9]. Together they form the SRP complex, which is recognized by the membrane bound SRP receptor Ftsy. Ftsy targets the SRP complex to the translocase complex [10]. In addition, it has been discovered that CsaA which seems to serve as a SecB homologue [11], can directly interact with SecA and precursor proteins to influence the secretory efficiency. (2) Translocase complex. In B. subtilis, the Sec translocases consist of SecA (motor protein) [12, 13], a heterotrimeric SecYEG complex (pore) [14-16], SecDF (chimeric protein) [17], YrbF (YajC homolgue) [18], and YidC homologues (SpoIIIJ and YqjG) [19]. The SecY, SecE and SecG proteins form the SecYEG complex. In general, the limited secretory efficiency is attributed to the insufficient capacity of the transport machinery. (3) Signal peptidases. In B. subtilis, five sip genes (sipT, sipS, sipU, sipV and sip W) for type I SPases have been identified. SipS and SipT are key to preprotein processing, while SipU, SipV and SipW appear to play minor roles in protein secretion. (4) Chaperones. $B$. subtilis has two types of molecular chaperones, intracellular and extracytoplasmic molecular chaperones. GroE and DnaK series are intracellular molecular chaperones mediating protein folding, minimizing aggregation and maintaining preproteins in translocation-competent conformations. The genes for these chaperones are organized in two operons, the groESL operon (groES-groEL) and the partial dnaK operon (grpE-dnaK-dnaJ). The extracytoplasmic folding factor PrsA is a well-defined lipoprotein required for subsequent folding of the mature protein into a stable and active conformation.

Although very high yields can be achieved with homologous proteins [20], production of heterologous proteins by $B$. subtilis is quite problematic [21]. In theory every step in the process of protein production and translocation can be a bottleneck causing reduced yields. As a result, it is imperative to explore the secretory process and engineer the secretory machinery components to improve the secretory efficiency. Recently, the overexpression of Ftsy and/or SRP components to improve protein secretion is described in a patent [22], as is the use of Pseudomonas glumea chaperones to improve the stability of the secretory proteins [23]. Diao constructed an artificial protein targeting pathway by co-expressing SecB (E. coli) and a B. subtilis hybrid SecA (the C-terminal 32 amino acids were replaced by the corresponding fragment of SecA from E. coli), and successfully increased the secretion of both MalE11 and PhoA [5]. Kakeshita deleted the C-terminal region of SecA, and the extracellular production of heterologous proteins was enhanced successfully in B. subtilis [13]. Mulder constructed an artificial secYEG operon to optimize their expression and substantially increased the secretory production of $\alpha$-amylase [14]. Nouaille complemented the Lactococcus lactis Sec machinery with SecDF from B. subtilis and the secretion of Staphylococcal Nuclease was obviously improved [24]. Malten had successfully increased the secretory production of recombinant protein by overexpressing the type I SPase in $B$. megaterium [25]. Wu reduced the formation of inclusion bodies and increased the secretory production yield of single chain antibody (SCA) in B. subtilis WB600, by coproducing GroE operon, Dnak operon and PrsA [26]. In many cases, overexpression of secreted proteins can cause jamming of membrane because of the shortage of Sec pathway components. The researches stated above notably suggest that modification or overexpression of Sec pathway components is an efficient approach for improving the secretory capacity of B. subtilis.

Although some specific components involved in Sec pathway were investigated, systematic studies of the effect of every component on heterologous protein secretion in B. subtilis are few. In this work, we described a systematic program to engineer the secretory machinery components to improve the secretory efficiency, namely: (1) effect of overexpression of every component involved in Sec pathway on heterologous protein secretion and identification of main bottlenecks in the secretory process in B. subtilis; (2) optimization of PrsA lipoprotein overexpression on expression level, providing the most efficient folding. (3) effect of PrsA overexpression in combination with other components screened out, respectively, on heterologous protein secretion. To reveal the bottlenecks of protein secretion process more convincingly, we chose two heterologous $\alpha$-amylases, AmyL ( $\alpha$-amylase from Bacillus licheniformis) and AmyS ( $\alpha$-amylase from Geobacillus stearothermophilus), as target proteins, which were secreted in distinctly different levels by $B$. subtilis. In this manner, we found that the deficiency of PrsA lipoprotein and chaperones of Dnak series is the most critical rate-limiting step in protein secretion. By overexpression of both intracellular and extracellular chaperones simultaneously, the enzyme activity and production of AmyL and AmyS were increased to approximately 9- and 
12-fold, correspondingly. Furthermore, the production of AmyL and AmyS by B. subtilis was conducted in $7.5 \mathrm{~L}$ fermentor with fed-batch strategy, respectively.

\section{Results}

Expression of recombinant $\alpha$-amylase AmyL and AmyS in B. subtilis

DNA fragment (Figure 1B) coding for thermostable $\alpha$-amylase AmyL or AmyS with their own signal peptide was obtained from B. licheniformis CICC 10181 or G. stearothermophilus ATCC 31195 by PCR and inserted into the E. coli/B. subtilis shuttle plasmid pMA5 (Figure 1A), under the control of the strong and constitutive promoter $\mathrm{P}_{\text {HpaII }}$ and upstream of the $\mathrm{T} 7$ terminator from $E$. coli. The obtained target plasmids pMA5L and pMA5S were transformed into B. subtilis 1A751, respectively, resulting into recombinant strains by selection at $37^{\circ} \mathrm{C}$ on the LB agar plates containing $50 \mu \mathrm{g} / \mathrm{mL}$ kanamycin and $1 \%$ soluble starch. The transformants with transparent rings around colonies were positive (Figure 1C). The insertion of AmyL or AmyS gene in the transformants was confirmed by PCR. The B. subtilis $1 \mathrm{~A} 751$ transformed with plasmid pMA5 was used as the control. The colonies were grown in $30 \mathrm{~mL}$ of SR medium at $37^{\circ} \mathrm{C}$ for $72 \mathrm{~h}$ in $250 \mathrm{~mL}$ shaker flasks. With the direction of signal peptide, mature AmyL and AmyS were released into extracellular medium. The $\alpha$-amylase activity of AmyL and AmyS in the culture supernatant was 90 and $111 \mathrm{U} / \mathrm{mL}$, respectively. The $\alpha$-amylase activity in the culture supernatant of the control strain was not detected under the same culture conditions. Meanwhile, SDS-PAGE analysis showed that a distinct and a slight bands with a molecular mass of about $55-\mathrm{kDa}$ which is in good agreement with the deduced value were observed in the medium fraction of AmyL producer and AmyS producer, respectively (Figure 1D). However, there was nearly no accumulation of AmyL and AmyS in the cell fraction. The results indicate that the $\mathrm{SP}_{a m y l}$ and $\mathrm{SP}_{a m y s}$ both have relatively high secretion efficiency. In addition, we can know that AmyL and AmyS are expressed in significantly different levels.

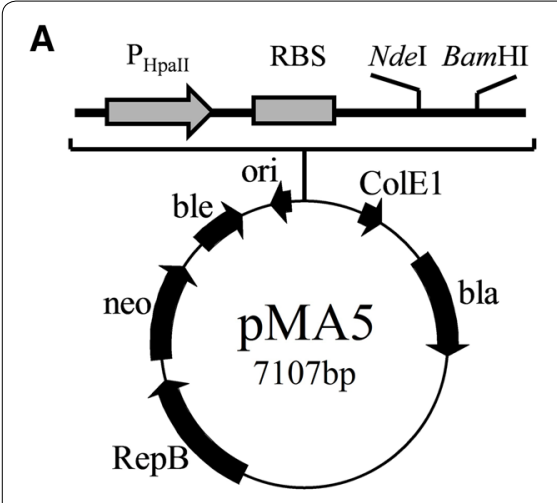

B
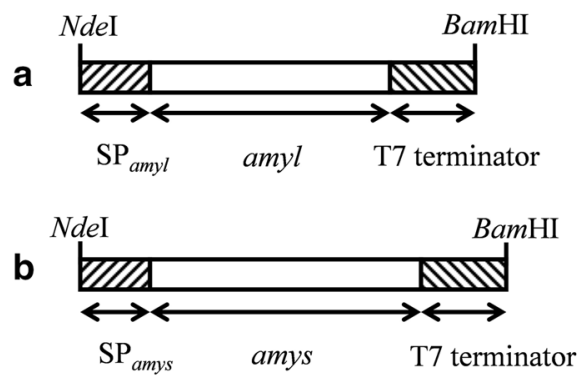

C

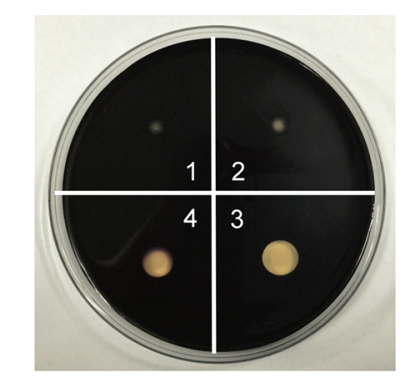

D

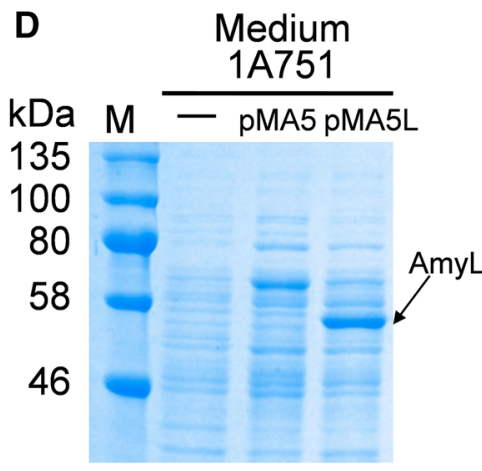

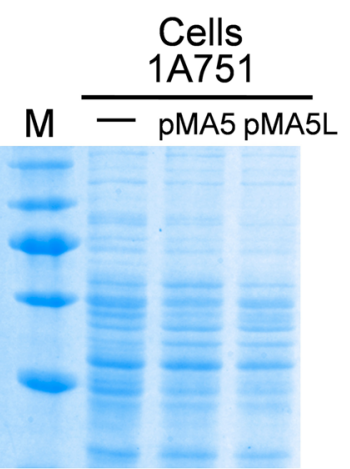

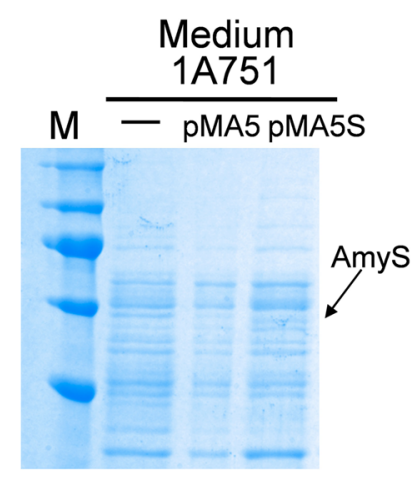

Medium

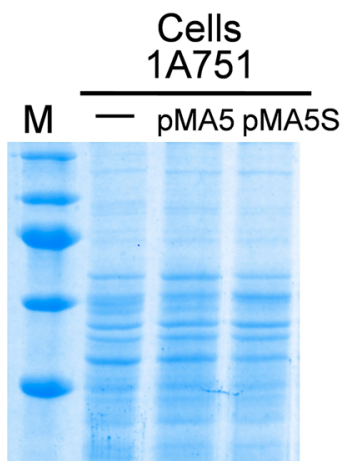

Figure 1 Expression and secretion of $\alpha$-amylase (AmyL and AmyS) in B. subtilis 1A751. A Vector map of E. coli/B. subtilis shuttle plasmid pMA5. P Hpall a widely used promoter from Staphylococcus aureus; RBS, ribosome binding site; ColE1, origin of replication for E. coli; bla, ampicillin resistance; RepB, origin of replication for $B$. subtilis; neo, kanamycin resistance. B The gene fragments of $\alpha$-amylase (AmyL and AmyS). Fragment a: AmyL encoding gene containing its native signal peptide $\mathrm{SP}_{\text {amyl }}$ plus T7 terminator; fragment b: AmyS encoding gene containing its native signal peptide SP ${ }_{\text {amys }}$ plus T7 terminator. C Starch hydrolysis of B. subtilis 1A751 (1), 1A751 (pMA5) (2), 1A751 (pMA5L) (3), 1A751 (pMA5S) (4). Strains were grown on LB agar medium containing $1 \%$ starch at $37^{\circ} \mathrm{C}$ overnight. Flood the surface of the plate with $5 \mathrm{~mL}$ Gram's iodine stain. D SDS-PAGE analysis of expression of $\alpha$-amylase (AmyL and AmyS) in medium and cell fractions by B. subtilis at incubation of $72 \mathrm{~h}$. Lane $\mathrm{M}$ molecular weight marker. 


\section{Development of an integration-vector system} for co-overexpression of two different target genes

To successfully increase a copy of the target genes or gene operons involved in Sec pathway on the chromosome in B. subtilis, we constructed an integration vector $\mathrm{pDDXG}$ (Additional file 1: Figure S1a), derivative from the vector pDD which contains $a m y E$ front and back regions for integration into the B. subtilis chromosome at $a m y E$ locus via double crossing-over. Firstly, we constructed the plasmid pDDX by inserting the strong and inducible promoter $\mathrm{P}_{x y l A}$ into the vector $\mathrm{pDD}$. As an upgrade, the integration vector pDDXG which could be used to overexpress two different genes simultaneously was constructed by inserting a strong and constitutive promoter $\mathrm{P}_{\text {grac }}$ into the vector $\mathrm{pDDX}$ downstream of the promoter $\mathrm{P}_{x y l A}$. To verify that the constructed $\mathrm{pDDXG}$ vector could fulfill the requirement, we constructed pDDXBG containing $b g a B$ gene under the control of the promoter $\mathrm{P}_{x y l A}$ and $\mathrm{pDDXGB}$ containing $b g a B$ gene under the control of the promoter $\mathrm{P}_{\text {grac }}$, and then these two AhdI-linearized plasmids were integrated into the chromosome of $B$. subtilis $1 \mathrm{~A} 751$ via double crossing-over, respectively. $\beta$-galactosidase activity was detected in both recombinant $1 \mathrm{~A} 751\left(\mathrm{P}_{x y l A}-\right.$ bgaB $)$ after induction by $2.0 \%$ xylose and 1A751 ( $\mathrm{P}_{\text {grac }}-$ bgaB $)$ without any induction (data not shown). This showed that pDDXG could be successfully used to overexpress one or two genes by integrating the target gene(s) into the chromosome.

\section{Effect of overexpression of single gene}

\section{involved in Sec pathway on $\alpha$-amylase secretion}

Overexpression of one or more secretory machinery components has been shown in a number of studies to assist the secretion and folding of several recombinant proteins $[14,25,27,28]$. To systematically investigate the impact of increased expression of the secretory machinery components on heterologous protein secretion in B. subtilis, 23 recombinant strains, 1A01 (ffh), 1A02 (hbs), 1A03 (scr), 1A04 (SRP), 1A05 (ftsy), 1A06 (csaA), 1A07 (secA), 1A08 (secY), 1A09 ( $\sec E), 1 \mathrm{~A} 10$ (secG), 1A11 (secYEG), 1A12 (secDF), 1A13 (yrbF), 1A14 (spoIIIJ), 1A15 (yqjG), 1A16 (sipT), 1A17 (sipS), 1A18 (sipU), 1A19 (sipV), 1A20 (sipW), 1A21 (groESL operon), 1A22 (partial dnaK operon) and 1A23 (prsA) (Table 1), were constructed by transforming different AhdI-linearized integration vectors (Table 2) containing corresponding genes or gene operons into B. subtilis 1A751. The growth of 23 recombinant strains in SR medium was similar to that of the parental strains 1A751. By real-time quantitative PCR, the transcriptional levels of the genes or gene operons involved in Sec pathway in corresponding recombinant strains were examined in the presence of $2.0 \%$ xylose so as to induce target genes expression. The respective mRNA levels of the 23 genes or gene operons in corresponding strains were several fold higher than that in $1 \mathrm{~A} 751$ (Figure 2a).

With construction of the 23 recombinant strains above, the effect of single gene involved in Sec pathway on the production and secretion of heterologous protein was studied systematically. The expression plasmids pMA5L and pMA5S were transformed into the 23 recombinant strains, respectively. The obtained strains were grown in liquid SR medium in the presence of $2.0 \%$ xylose, and the $\alpha$-amylase activity of AmyL and AmyS in medium of the corresponding recombinant strains was measured compared with the parental strains $1 \mathrm{~A} 751$ at $72 \mathrm{~h}$. For AmyL production (Figure $2 \mathrm{~b}$ ), overexpression of $\operatorname{prs} A$ resulted in a significant increase (3.2-fold) of the $\alpha$-amylase activity, and overexpression of $\sec D F, S R P$, partial dnaK operon, ftsy, sipS and secYEG resulted in moderate or marginal increase $(147,138,133,132,122$ and 108\%, respectively; $t$ test, all $\mathrm{P}<0.05)$ of the $\alpha$-amylase activity. However, with the overexpression of other genes, the $\alpha$-amylase activity was not improved and even reduced. As to AmyS production (Figure 2c), more remarkable improvement (5.5-fold) of the $\alpha$-amylase activity was observed when prs $A$ was overexpressed, and slight enhancements (149, $138,130,127,126,125,124$ and $112 \%$, respectively; $t$ test, all $\mathrm{P}<0.05)$ were obtained when partial dnaK operon, $\sec D F, \sec A, \operatorname{sip} T$, groESL operon, ftsy, SRP and sipS were overexpressed. Similarly, with the overexpression of other genes, the $\alpha$-amylase activity was also not improved and even reduced. These findings suggest that overexpression of prsA, secDF, SRP, partial dnaK operon, ftsy, sipS, $\sec Y E G$, secA or sipT, more or less, may enhance the production of $\alpha$-amylase in $B$. subtilis. Above all, $\operatorname{prs} A$ is the most vital rate-limiting factor for $\alpha$-amylase production and secretion in the Sec pathway.

\section{Optimization of PrsA overexpression level in B. subtilis}

PrsA is a lipoprotein that consists of a $33-\mathrm{kDa}$ lysinerich protein part and the $\mathrm{N}$-terminal cysteine with a thiol-linked diacylglycerol anchoring the protein to the outer leaflet of the cytoplasmic membrane $[29,30]$. The PrsA lipoprotein is crucial for efficient secretion of a number of exoproteins. Researches show that there is a linear correlation between the number of cellular PrsA molecules and the number of secreted AmyQ molecules over a wide range of $p r s A$ and amyQ expression levels [31]. Meanwhile, our result stated above suggests that PrsA expression level is rate limiting in the secretion of AmyL and AmyS. To further investigate the relationship between $\alpha$-amylase (AmyL and AmyS) secretion and PrsA expression and enhance the production and secretion of AmyL and AmyS, PrsA overexpression level was optimized. 
Table 1 Strains used in this study

\begin{tabular}{|c|c|c|}
\hline Strains & Genotype and/or relevant characteristic(s) & Source \\
\hline E. coli DH5 $\alpha$ & $\mathrm{F}^{-} \Delta$ lacU169(Ø80d lacZ $\left.\Delta \mathrm{M} 15\right)$ supE44 hsdR17 recA1 gyrA96 endA1 thi-1 relA1 & Invitrogen \\
\hline B. licheniformis CICC 10181 & Wild-type B. licheniformis, amyl gene & $\mathrm{CICC}$ \\
\hline G. stearothermophilus ATCC 31195 & Wild-type G. stearothermophilus, amys gene & ATCC \\
\hline B. subtilis 1 A751 & eg/S $\Delta 102$ bg/T/bg/S $\Delta$ EV aprE nprE his & BGSC \\
\hline $1 \mathrm{AXBG}$ & 1A751 with integration of pDDXBG (amyE::P $\left.P_{x y / A}-b g a B\right) ; \mathrm{Cm}^{r}$ & This work \\
\hline $1 \mathrm{AXGB}$ & 1A751 with integration of pDDXGB (amyE::.P grac $-b g a B) ; \mathrm{Cm}^{r}$ & This work \\
\hline $1 \mathrm{~A} 01$ & 1A751 with integration of pDD01 (amyE::P $\left.P_{x y / A}-f f h\right) ; \mathrm{Cm}^{r}$ & This work \\
\hline $1 \mathrm{~A} 02$ & 1A751 with integration of pDD02 (amyE::P $\left.{ }_{x y l A}-h b s\right) ; \mathrm{Cm}^{r}$ & This work \\
\hline $1 \mathrm{~A} 03$ & 1A751 with integration of pDD03 (amyE::P $\left.P_{x y / A}-s c r\right) ; \mathrm{Cm}^{r}$ & This work \\
\hline $1 \mathrm{~A} 04$ & 1A751 with integration of pDD04 (amyE::P $\left.P_{x y / A}-S R P\right) ; \mathrm{Cm}^{r}$ & This work \\
\hline $1 \mathrm{~A} 05$ & 1A751 with integration of pDD05 (amyE::P $\left.x y / A^{-f t s y}\right) ; \mathrm{Cm}^{r}$ & This work \\
\hline $1 \mathrm{~A} 06$ & 1A751 with integration of pDD06 (amyE::P $\left.P_{x y / A}-C s a A\right) ; \mathrm{Cm}^{r}$ & This work \\
\hline $1 \mathrm{~A} 07$ & 1A751 with integration of pDD07 (amyE::P $\left.P_{x y / A}-\sec A\right) ; \mathrm{Cm}^{r}$ & This work \\
\hline $1 \mathrm{~A} 08$ & 1A751 with integration of pDD08 (amyE::P $\left.P_{x y l A}-\sec \right) ; \mathrm{Cm}^{r}$ & This work \\
\hline $1 \mathrm{~A} 09$ & 1A751 with integration of pDD09 (amyE::P $\left.P_{x y / A}-\sec E\right) ; \mathrm{Cm}^{r}$ & This work \\
\hline $1 \mathrm{~A} 10$ & 1A751 with integration of pDD10 (amyE::P $\left.P_{x y / A}-\sec G\right) ; \mathrm{Cm}^{r}$ & This work \\
\hline $1 \mathrm{~A} 11$ & 1A751 with integration of pDD11 (amyE::P $\left.P_{x y / A}-\sec Y E G\right) ; \mathrm{Cm}^{r}$ & This work \\
\hline $1 \mathrm{~A} 12$ & 1A751 with integration of pDD12 (amyE::P $\left.\mathrm{x}_{x y / A}-\sec D F\right) ; \mathrm{Cm}^{\mathrm{r}}$ & This work \\
\hline $1 \mathrm{~A} 13$ & 1A751 with integration of pDD13 (amyE::P $\left.P_{x y / A}-y r b F\right) ; \mathrm{Cm}^{r}$ & This work \\
\hline $1 \mathrm{~A} 14$ & 1A751 with integration of pDD14 (amyE::P $\left.P_{x y / A}-s p o l l I J\right) ; \mathrm{Cm}^{r}$ & This work \\
\hline $1 \mathrm{~A} 15$ & 1A751 with integration of pDD15 (amyE::P $\left.P_{x y / A}-y q j G\right) ; C m^{r}$ & This work \\
\hline $1 \mathrm{~A} 16$ & 1A751 with integration of pDD16 (amyE::P $\left.{ }_{x y / A}-\operatorname{sip} T\right) ; \mathrm{Cm}^{r}$ & This work \\
\hline $1 \mathrm{~A} 17$ & 1A751 with integration of pDD17 (amyE::P $\left.P_{x y / A}-\operatorname{sipS}\right) ; \mathrm{Cm}^{r}$ & This work \\
\hline $1 \mathrm{~A} 18$ & 1A751 with integration of pDD18 (amyE::P $\left.P_{x y / A}-\operatorname{sip} U\right) ; \mathrm{Cm}^{r}$ & This work \\
\hline $1 \mathrm{~A} 19$ & 1A751 with integration of pDD19 (amyE::P $x y / A^{-s i p V) ; ~} \mathrm{Cm}^{r}$ & This work \\
\hline $1 \mathrm{~A} 20$ & 1A751 with integration of pDD20 (amyE::P $x y / A^{-s i p W) ; ~} \mathrm{Cm}^{r}$ & This work \\
\hline $1 \mathrm{~A} 21$ & 1A751 with integration of pDD21 (amyE::.P $P_{x y / A}$-groESL operon); $\mathrm{Cm}^{r}$ & This work \\
\hline $1 \mathrm{~A} 22$ & 1A751 with integration of pDD22 (amyE::P $P_{x y / A}$-partial dnaK operon); $\mathrm{Cm}^{r}$ & This work \\
\hline $1 \mathrm{~A} 23$ & 1A751 with integration of pDD23 (amyE::. $\left.P_{x y l A}-p r s A\right) ; \mathrm{Cm}^{r}$ & This work \\
\hline $1 \mathrm{~A} 231$ & 1A751 with integration of pDD231 (amyE:.:P xylA - prsA, $\left.P_{\text {grac }}-S R P\right) ; \mathrm{Cm}^{r}$ & This work \\
\hline $1 \mathrm{~A} 232$ & 1A751 with integration of pDD232 (amyE:::P xy/A-prsA, $\left.P_{\text {grac }}-\mathrm{ftsy}\right) ; \mathrm{Cm}^{r}$ & This work \\
\hline $1 \mathrm{~A} 233$ & 1A751 with integration of pDD233 (amyE::.P ${ }_{x y l A}-$ prsA, $\left.P_{\text {grac }}-\sec A\right) ; \mathrm{Cm}^{r}$ & This work \\
\hline $1 \mathrm{~A} 234$ & 1A751 with integration of pDD234 (amyE::P $P_{x y / A}-$ prsA, $P_{\text {grac }}-$ sec $\left.Y E G\right) ; \mathrm{Cm}^{r}$ & This work \\
\hline $1 \mathrm{~A} 235$ & 1A751 with integration of pDD235 (amyE::P xylA $\left.^{-p r s A}, P_{\text {grac }}-\sec D F\right) ; \mathrm{Cm}^{r}$ & This work \\
\hline $1 \mathrm{~A} 236$ & 1A751 with integration of pDD236 (amyE::P $\mathrm{PyIA}_{\mathrm{A}}-$ prsA, $\mathrm{P}_{\text {grac }}$-groESL operon); $\mathrm{Cm}^{r}$ & This work \\
\hline $1 \mathrm{~A} 237$ & 1A751 with integration of pDD237 (amyE::P $x y / A-p r s A, P_{\text {grac }}-$ partial dnaK opeon); $\mathrm{Cm}^{r}$ & This work \\
\hline $1 \mathrm{~A} 238$ & 1A751 with integration of pDD238 (amyE::.P $\left.\mathrm{xylA}_{\mathrm{A}}-\mathrm{prs} A, \mathrm{P}_{\text {grac }}-\mathrm{sip} T\right) ; \mathrm{Cm}^{\mathrm{r}}$ & This work \\
\hline $1 \mathrm{~A} 239$ & 1A751 with integration of pDD239 (amyE::P $\mathrm{xylA}_{\mathrm{A}}$-prsA, $\mathrm{P}_{\text {grac }}-$ sipS); $\mathrm{Cm}^{r}$ & This work \\
\hline
\end{tabular}

CICC China Center of Industrial Culture Collection (http://www.chinacicc.org), ATCC American Type Culture Collection, BGSC Bacillus Genetic Stock Center, USA.

The recombinant strains $1 \mathrm{~A} 23$ (pMA5L) and $1 \mathrm{~A} 23$ (pMA5S) secreted constitutively AmyL and AmyS, respectively, and their additional prsA has been placed under the control of the promoter $\mathrm{P}_{x y l A}$ at amyE locus in chromosome (see Table 1 and "Methods"). 1A23 (pMA5L) and 1A23 (pMA5S) were cultivated in the presence of xylose of different concentrations [0, 0.5, 1.0, 1.5, 2.0, 2.5, 3.0, 3.5, 4.0, 4.5 and $5.0 \%(\mathrm{w} / \mathrm{v})]$. The addition of xylose within a suitable concentration range greatly enhanced the $\alpha$-amylase activity of both AmyL and AmyS, and the maximal $\alpha$-amylase activity of AmyL and AmyS in the medium were obtained when $4.0 \%$ xylose was added (Figure 3). The result showed that $\alpha$-amylase secretion was dependent very closely on PrsA expression level whether expressed at a high level (AmyL) or at a low level (AmyS). 
Table 2 Plasmids used in this study

\begin{tabular}{|c|c|c|}
\hline Plasmids & Genotype and/or relevant characteristic(s) & Source \\
\hline pET28-a(+) & $\begin{array}{l}\text { E. coli expression plasmid, } \mathrm{T} 7 \text { promoter, } \mathrm{T} 7 \\
\text { terminator, Kan }\end{array}$ & Lab stock \\
\hline pET28L & pET28-a(+) derivative, amyl & This work \\
\hline pET28S & pET28-a(+) derivative, amys & This work \\
\hline pMA5 & E. coli/B. subtilis shuttle vector, $\mathrm{P}_{\text {Hpall }}, \mathrm{Ap}^{r}, \mathrm{Km}^{r}$ & BGSC \\
\hline pMA5L & pMA5 derivative, $\mathrm{SP}_{\text {amyl }}$ amyl, $\mathrm{T} 4$ terminator & This work \\
\hline pMA5S & pMA5 derivative, $\mathrm{SP}_{\text {amys' }}$ amys, $\mathrm{T} 4$ terminator & This work \\
\hline pHCMCO4 & $P_{x y / A r} A^{r}, \mathrm{Cm}^{r}$ & Lab stock \\
\hline pHT43 & $P_{\text {grac }} \mathrm{SP}_{\text {amy }}, \mathrm{Ap}^{r}, \mathrm{Cm}^{r}$ & Lab stock \\
\hline pDD & Integration vector, $\mathrm{pDL}$ derivative, $\mathrm{Ap}^{r}, \mathrm{Cm}^{r}$ & This work \\
\hline pDDX & pDD derivative, $P_{x y / A}$ & This work \\
\hline pDDXG & pDDX derivative, $P_{x y \mid A}, P_{\text {grac }}$ & This work \\
\hline pDDXBG & pDDXG derivative, $b g a B$ & This work \\
\hline pDDXGB & pDDXG derivative, $b g a B$ & This work \\
\hline pDD01 & pDDXG derivative, ffh & This work \\
\hline pDD02 & pDDXG derivative, $h b s$ & This work \\
\hline pDD03 & pDDXG derivative, scr & This work \\
\hline pDD04 & pDDXG derivative, $S R P$ & This work \\
\hline pDD05 & pDDXG derivative, ftsy & This work \\
\hline pDD06 & pDDXG derivative, csaA & This work \\
\hline pDD07 & pDDXG derivative, sec $A$ & This work \\
\hline pDD08 & pDDXG derivative, sec $Y$ & This work \\
\hline pDD09 & pDDXG derivative, secE & This work \\
\hline pDD10 & pDDXG derivative, secG & This work \\
\hline pDD11 & pDDXG derivative, secYEG & This work \\
\hline pDD12 & pDDXG derivative, secDF & This work \\
\hline pDD13 & pDDXG derivative, yrbF & This work \\
\hline pDD14 & pDDXG derivative, spollIJ & This work \\
\hline pDD15 & pDDXG derivative, yqjG & This work \\
\hline pDD16 & pDDXG derivative, sipT & This work \\
\hline pDD17 & pDDXG derivative, sipS & This work \\
\hline pDD18 & pDDXG derivative, sipU & This work \\
\hline pDD19 & pDDXG derivative, sipV & This work \\
\hline pDD20 & pDDXG derivative, sipW & This work \\
\hline pDD21 & pDDXG derivative, groESL operon & This work \\
\hline pDD22 & pDDXG derivative, partial dnaK operon & This work \\
\hline pDD23 & pDDXG derivative, prs $A$ & This work \\
\hline pDD231 & pDD23 derivative, prsA, SRP & This work \\
\hline pDD232 & pDD23 derivative, prsA, ftsy & This work \\
\hline pDD233 & pDD23 derivative, prsA, sec $A$ & This work \\
\hline pDD234 & pDD23 derivative, $\operatorname{pr} A$, secYEG & This work \\
\hline pDD235 & pDD23 derivative, $\operatorname{prs} A$, secDF & This work \\
\hline pDD236 & pDD23 derivative, prsA, groESL operon & This work \\
\hline pDD237 & pDD23 derivative, prsA, partial dnaKoperon & This work \\
\hline pDD238 & pDD23 derivative, prsA, sipT & This work \\
\hline pDD239 & pDD23 derivative, prsA, sipS & This work \\
\hline
\end{tabular}

\section{Combinatorial overexpression of PrsA combined with the} screened components

Following identification of PrsA as a main engineering target and the optimization of PrsA overexpression level, we next undertook the combinatorial overexpression of pairs of Sec pathway genes in order to unveil further bottlenecks of heterologous protein production and secretion. For this goal, nine genes or gene operons SRP, ftsy, secA, secYEG, secDF, groESL operon, partial dnaK operon, sipT and sipS, overexpression of which slightly enhanced the production and secretion of AmyL or AmyS, were screened to be overexpressed combined with PrsA, respectively. Therefore, we generated nine double-overexpression strains as follows: 1A231 (prsA SRP), 1A232 (prsA ftsy), 1A233 (prsA secA), 1A234 (prsA secYEG), 1A235 (prsA secDF), $1 \mathrm{~A} 236$ (prsA groESL opeon), 1A237 (prsA partial dnaK operon), 1A238 (prsA sipT), 1A239 (prsA sipS), by transforming AhdI-lineared corresponding integration vectors into $1 \mathrm{~A} 751$. The nine genes or gene operons were all under the control of $\mathrm{P}_{\text {grac }}$, and the prs $A$ gene was still under the control of $\mathrm{P}_{x y l A}$. By real-time quantitative $\mathrm{PCR}$, the transcriptional levels of $\operatorname{prs} A$ (with the addition of $4.0 \%$ xylose) and other nine screened genes in corresponding recombinant strains were much higher than that in control strain 1A751 (Figure 4a).

The expression plasmids pMA5L and pMA5S were transformed into the nine recombinant strains, respectively. The obtained strains were grown in liquid SR medium in the presence of $4.0 \%$ xylose, and the $\alpha$-amylase activity of AmyL and AmyS in medium of corresponding recombinant strains were measured compared with the strain 1A23 (pMA5L or pMA5S) at $72 \mathrm{~h}$ (Figure 4b). For AmyL production, the overexpression of prsA combined with partial dnaK operon, secDF, ftsy and sipT, respectively, improved the activity of $\alpha$-amylase in medium in different extent (160, 131, 108 and 108\%, respectively; $t$ test, all $\mathrm{P}<0.05)$. As to AmyS production, the overexpression of prsA combined with partial dnaK operon, ftsy and sipT respectively, resulted in moderate increase $(173,151$ and $132 \%$, respectively; $t$ test, all $\mathrm{P}<0.05)$ of $\alpha$-amylase in medium. It can be seen that when prs $A$ was overexpressed combined with partial dnaK operon, the $\alpha$-amylase activity of AmyL and AmyS in medium both were the highest, indicating that the chaperones of Dnak series play a vital role in $\alpha$-amylase production.

To provide further evidence supporting the results described above, the SDS-PAGE analysis was performed to compare the production of $\alpha$-amylase. Distinct bands with 


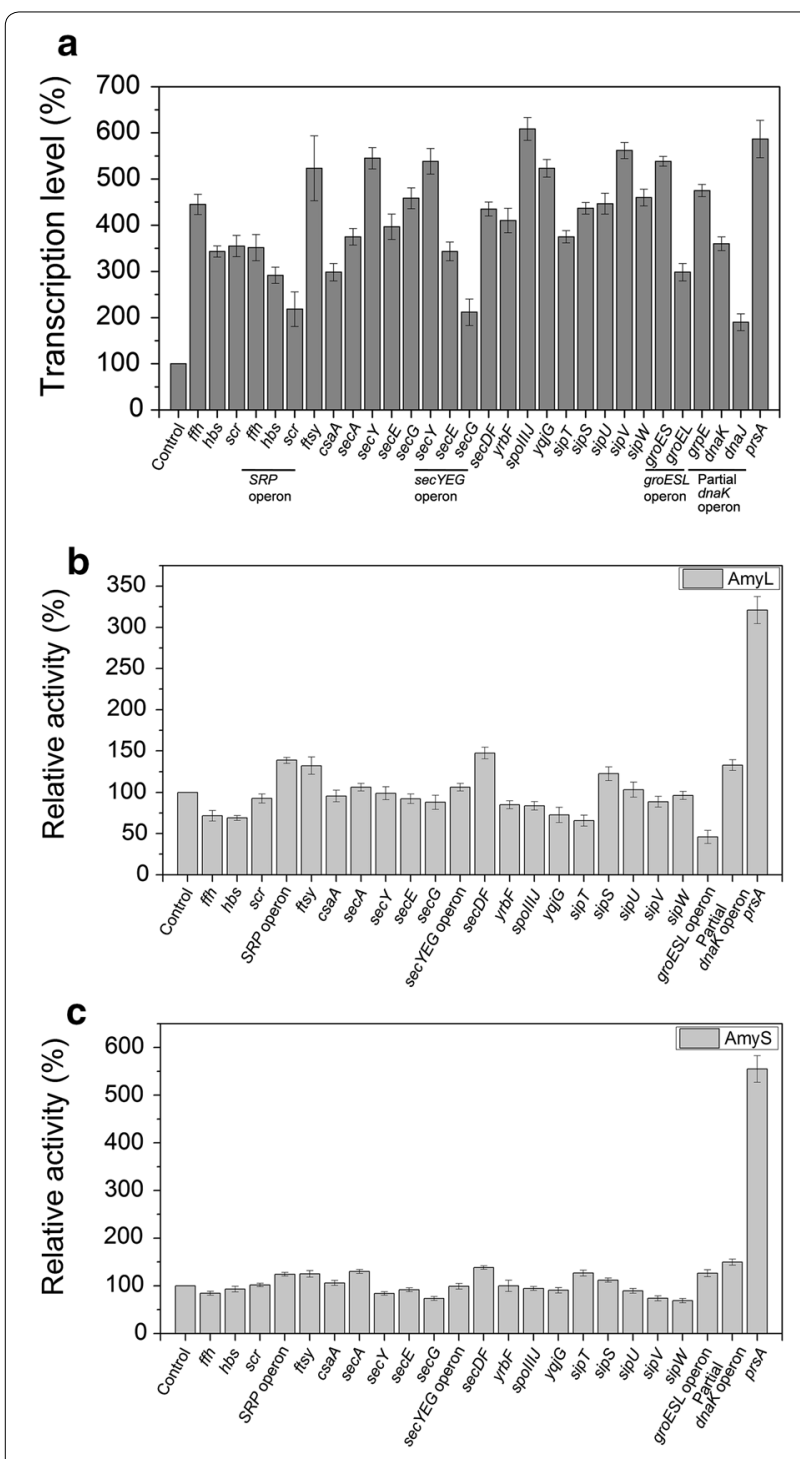

Figure 2 Overexpression of single component involved in Sec pathway in B. subtilis. a The transcriptional levels of 23 genes or gene operons in 23 corresponding recombinant strains, respectively. Column Control represents the transcriptional levels of the 23 genes or gene operons in 1A751 (all regarded as 100\%). b Effect of overexpression of single gene or gene operon on AmyL secretion. Column Control is the $\alpha$-amylase activity of 1 A751 (pMA5L), regarded as $100 \%$. c Effect of overexpression of single gene or gene operon on AmyS secretion. Column Control is the $\alpha$-amylase activity of 1A751 (pMA5S), served as $100 \%$. The samples were collected at incubation of $72 \mathrm{~h}$ in $250 \mathrm{~mL}$ shake-flask and centrifuged at $10,000 \times \mathrm{g}$ and $4^{\circ} \mathrm{C}$ for $10 \mathrm{~min}$. After extraction, the supernatant was used for analysis of $\alpha$-amylase activity. The $\alpha$-amylase activity was converted to percent increase referring to the respective control strains. Data represent the mean of three parallel experiments, and error bars represent standard error. Specifically, the genes or gene operons ffh, hbs, scr, SRP, ftsy, csaA, secA, secY, secE, secG, secYEG, secDF, yrbF, spollIJ, yqjG, sipT, sipS, sipU, sipV, sipW, groESL operon, partial dnaK operon and prsA in $\mathbf{a}, \mathbf{b}$ and $\mathbf{c}$ corresponded to strains 1A01, 1A02, 1A03, 1A04, 1A05, 1A06, 1A07, 1A08, 1A09, 1A10, 1A11, 1A12, 1A13, 1A14, 1A15, 1A16, 1A17, 1A18, $1 \mathrm{~A} 19,1 \mathrm{~A} 20,1 \mathrm{~A} 21,1 \mathrm{~A} 22$ and $1 \mathrm{~A} 23$, respectively. a molecular mass of about $55 \mathrm{kDa}$ were observed which was in good agreement with the deduced value in both AmyL and AmyS production strains, which was consistent with the results as previously mentioned (Figure 4c).

\section{Characterization of engineered vs. parental strains on $\alpha$-amylase production}

We chose recombinant strain 1A237 overexpressing prsA and partial $d n a K$ operon for detailed analyses on growth condition and $\alpha$-amylase (AmyL and AmyS) production, and, meanwhile, compared them with the parental strain 1A751. Whether producing AmyL or AmyS, 1A237 and 1A751 have similar biomass during the early stage of exponential growth $(<12 \mathrm{~h})$; however, they differed significantly upon entry into the late stage of exponential growth (Figure 5a). The $\mathrm{OD}_{600}$ values of $1 \mathrm{~A} 237$ peaked at $24 \mathrm{~h}$ and were lower than that of $1 \mathrm{~A} 751$, which continued to increase after $12 \mathrm{~h}$ and peaked at $36 \mathrm{~h}$. Nevertheless, simultaneous overexpression of prsA and dnaK operon improved the final $\alpha$-amylase activity of AmyL and AmyS by about ninefold and 12 -fold (Figure $5 \mathrm{~b}$ ). The results of SDS-PAGE analysis of $\alpha$-amylase in medium were consistent with the activity data (Figure 5d). Furthermore, B. subtilis 1A237 had much higher productivity of AmyL and AmyS (approximately 13- and 17-fold, respectively) than that of the parental strain 1A751 (Figure 5c).

As shown above, AmyS producer exhibited generally greater increases of yield compared to AmyL producer, indicating that this, the protein of initially lower production, has more room for phenotype improvement. In addition, phenotypic characterization of engineered $\alpha$-amylase producers revealed that higher $\alpha$-amylase production was accompanied by reduced growth rates. Specifically, growth rates were determined as $\mu=0.30 \mathrm{~h}^{-1}$ for 1A751 (pMA5-AmyL), $\mu=0.23 \mathrm{~h}^{-1}$ for 1A237 (pMA5-AmyL), $\mu=0.29 \mathrm{~h}^{-1}$ for 1A751 (pMA5-AmyS), and $\mu=0.21 \mathrm{~h}^{-1}$ for 1A237 (pMA5-AmyS). The negative impact of $\alpha$-amylase production on the growth rate was also observed in various other engineered strains of this study (data not shown).

\section{Production of $\alpha$-amylase (AmyL and AmyS) in $7.5 \mathrm{~L}$ fermentor with fed-batch strategy}

The expression efficiency of B. subtilis 1A237 (pMA5AmyL) and 1A237 (pMA5-AmyS) were further explored in $7.5 \mathrm{~L}$ fermentor, respectively. The fermentor was inoculated with $5 \%(\mathrm{v} / \mathrm{v})$ of freshly cultured 1A237 (pMA5AmyL) or 1A237 (pMA5-AmyS) grown in SR medium at $37^{\circ} \mathrm{C}$ for $18 \mathrm{~h}$. To maintain cell growth and $\alpha$-amylase production, we choose a fed-batch strategy. When the cell growth rate was constant, $8.0 \%(\mathrm{w} / \mathrm{v})$ soluble starch was added at a constant flow rate until the final concentration of soluble starch was up to $4.0 \%(w / v)$. For AmyL 

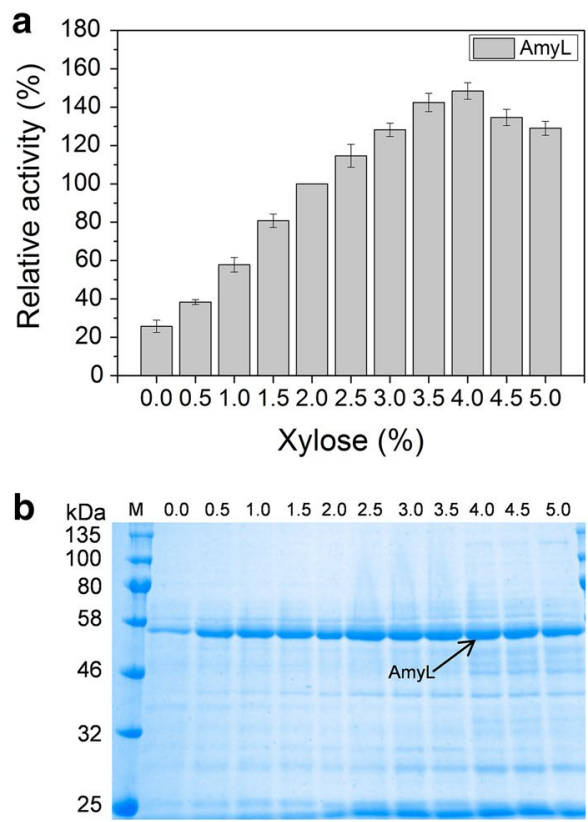
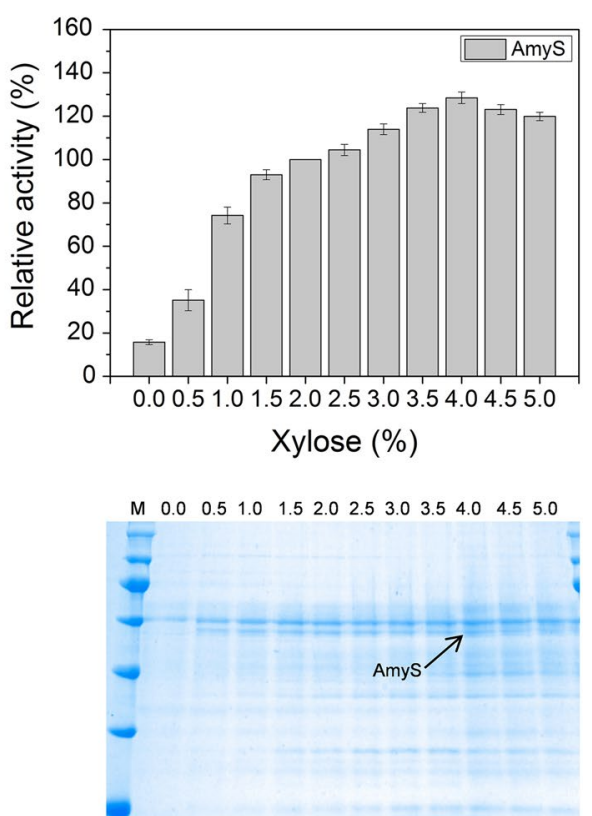

Figure 3 Optimization of PrsA overexpression level in B. subtilis. 1A23 (pMA5L) and 1A23 (pMA5S) were grown in SR medium supplemented with xylose of different concentrations $[0,0.5,1.0,1.5,2.0,2.5,3.0,3.5,4.0,4.5$ and $5.0 \%(\mathrm{w} / \mathrm{V})]$. a Analysis of $\alpha$-amylase (AmyL and AmyS) activity in the supernatant at incubation of $72 \mathrm{~h}$. The $\alpha$-amylase activity was converted to percent increase referring to $2.0 \%$ xylose. $\mathbf{b}$ SDS-PAGE analysis of $\alpha$-amylase (AmyL and AmyS) distribution in culture supernatants.

production (Figure 6a), during the growth phase, the maximum biomass in the fermentor reached $41.3\left(\mathrm{OD}_{600}\right)$ at $24 \mathrm{~h}$. The activity of $\alpha$-amylase in medium was continuously increased and reached the maximum of $1,352 \mathrm{U} /$ $\mathrm{mL}$ at $84 \mathrm{~h}$ with a high productivity of $16.1 \mathrm{U} / \mathrm{mL}$ h. For AmyS production (Figure $6 \mathrm{~b}$ ), the maximum biomass in the fermentor reached $32.3\left(\mathrm{OD}_{600}\right)$ at $36 \mathrm{~h}$. The activity of $\alpha$-amylase in medium was continuously increased and reached the maximum of $2,300 \mathrm{U} / \mathrm{mL}$ at $84 \mathrm{~h}$ with a high productivity of $27.4 \mathrm{U} / \mathrm{mL}$ h. The high activity of $\alpha$-amylase indicated that $B$. subtilis was a suitable host for the industrial production of $\alpha$-amylase.

\section{Discussion}

In the present work, to produce two heterologous proteins (AmyL and AmyS) in B. subtilis, amyl and amys were equipped with their native signal peptide $\mathrm{SP}_{a m y l}$ and $\mathrm{SP}_{a m y S}$, respectively, and under the control of the promoter $\mathrm{P}_{\text {HpaII }}$. $\mathrm{SP}_{a m y l}$ and $\mathrm{SP}_{a m y s}$ are Sec-dependent signal peptides, so that AmyL and AmyS can traverse the cytoplasmic membrane via the Sec-translocon. $\mathrm{P}_{\text {HpaII }}$ is a widely used, strong and constitutive promoter from Staphylococcus aureus. The recombinant AmyL was secreted into the culture medium by B. subtilis 1A751 in a markedly higher expression level compared with AmyS (Figure 1c). Whereas, there was no AmyL or AmyS accumulation in the cell fraction, which indicates that nearly the whole AmyL or AmyS can be directed into the medium and $\mathrm{SP}_{a m y l}$ and $\mathrm{SP}_{a m y s}$ are efficient signal peptides for AmyL and AmyS secretion, respectively. However, the $\alpha$-amylase activity of AmyL or AmyS in 1A751 was still low, compared to some public studies [32, 33]. Researches show that high gene expression level may result in saturation of the Sec-translocon capacity; that is to say, the expression level of the components involved in Sec pathway may be insufficient for secretion of heterologous proteins. To improve the yields of heterologous proteins, it is necessary to identify the bottlenecks hampering their production.

Sec pathway is the major route for protein secretion in B. subtilis. The components involved in Sec pathway are roughly divided into four categories: Signal recognition particle, translocase complex, signal peptidases and chaperones. We evaluated the single overexpression of 23 genes or gene operons involved in Sec pathway on the production of AmyL and AmyS, respectively, and found that prsA overexpression markedly improved the production of both AmyL (3.2-fold) and AmyS (5.5fold) (Figure 2). The results point to PrsA as a bottleneck for the secretion of AmyL and AmyS, which is consistent with previous studies [31, 34]. PrsA is an extracellular folding chaperone that influences protein folding in the (pseudo) periplasmic space. In this environment the lipoprotein PrsA is required for protein stability 
a

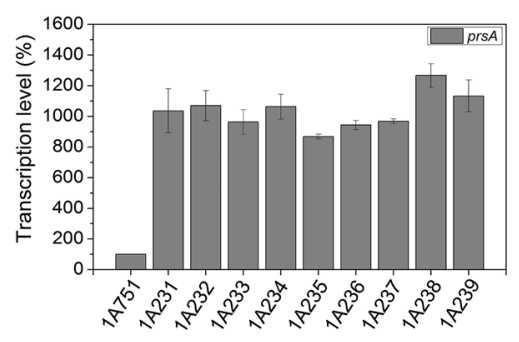

b
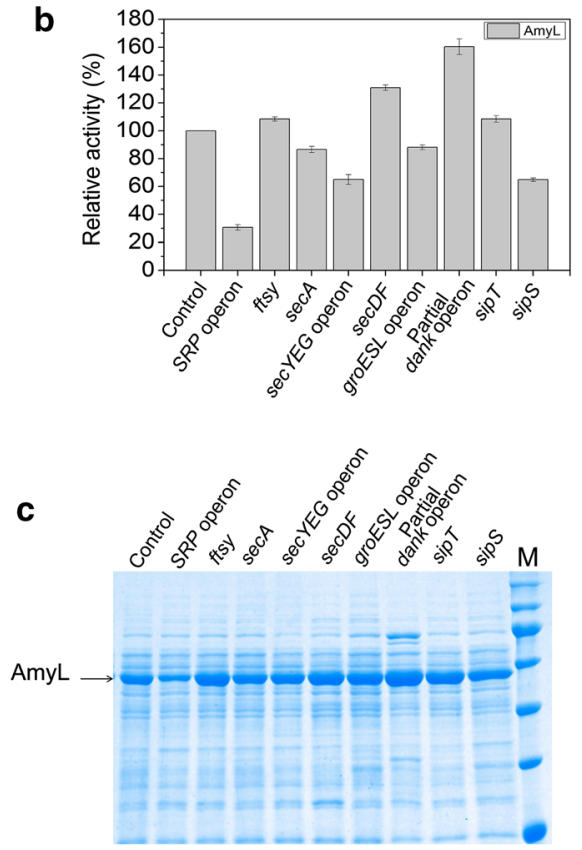
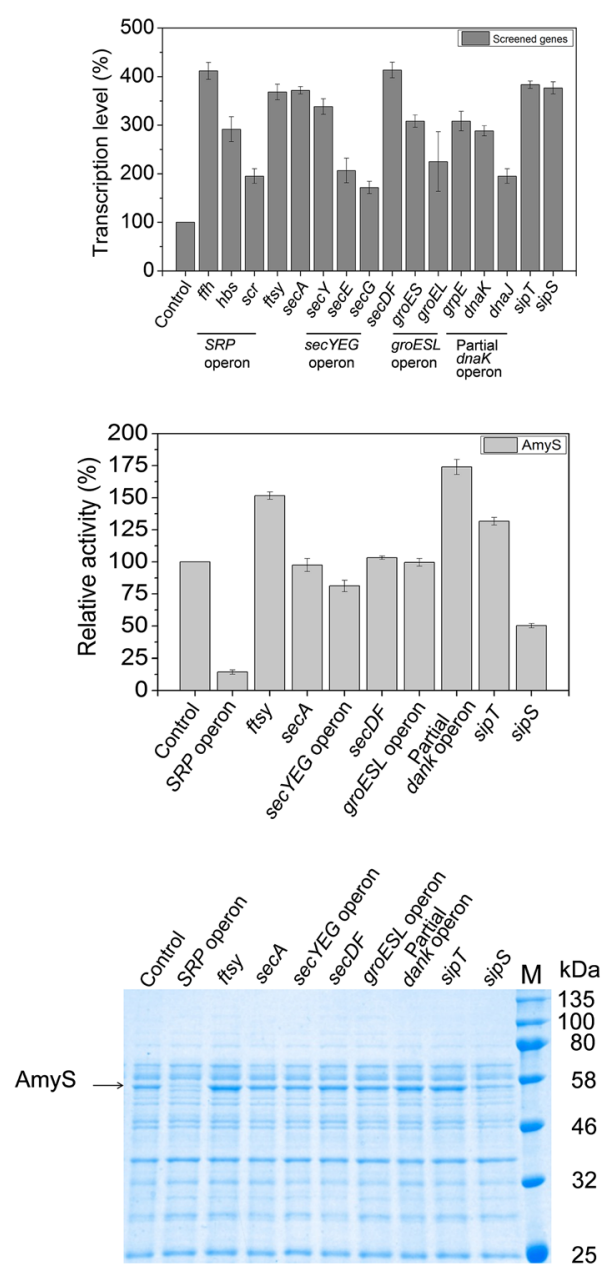

Figure 4 Overexpression of PrsA combined with the screened components in B. subtilis. a The transcriptional levels of the genes or gene operons in B. subtilis. The first figure shows the transcriptional level of prs $A$ in recombinant strains; the transcription level of $1 \mathrm{~A} 751$ (control) is regarded as $100 \%$. The second figure indicates the transcriptional levels of nine genes or gene operons in nine corresponding strains, respectively; Control, the transcriptional levels of nine genes or gene operons (all regarded as 100\%) in 1A751. b Analysis of $\alpha$-amylase (AmyL and AmyS) activity in nine combinational overexpression strains. The $\alpha$-amylase activity was converted to percent increase referring to the respective control strains [the former, 1 A23 (pMA5L); the latter, 1A23 (pMA5S)]. c SDS-PAGE analysis of $\alpha$-amylase (AmyL and AmyS) in nine combinational overexpression strains. Lane M molecular weight marker. The former Control is 1A23 (pMA5L); the latter Control is 1A23 (pMA5S). Specifically, the genes or gene operons SRP, ftsy, secA, secYEG, secDF, groESL operon, partial dnaK operon, sipT and sipS in $\mathbf{a}, \mathbf{b}$ and $\mathbf{c}$ corresponded to strains $1 \mathrm{~A} 231,1 \mathrm{~A} 232,1 \mathrm{~A} 233,1 \mathrm{~A} 234,1 \mathrm{~A} 235$, $1 \mathrm{~A} 236,1 \mathrm{~A} 237,1 \mathrm{~A} 238$ and $1 \mathrm{~A} 239$, respectively.

in the post-translocational phase of secretion [31, 34, 35]. AmyL and AmyS are heterologous proteins from $B$. licheniformis and B. stearothermophilus, respectively. With the overexpression of $p r s A$, more $\alpha$-amylase (AmyL or AmyS) was rapidly folded into its native conformation, allowing $\alpha$-amylase to gain resistance against extracellular proteases. In addition, the result that AmyL expressed at relative high level and AmyS expressed at relative low level were both significantly enhanced by overexpression of prsA was consistent with previous studies [31]. On account of the key role of PrsA in the process of protein secretion, we modulated the expression levels of prs $A$.
With the increase of xylose concentration, the expression level of prsA was improved. The $\alpha$-amylase activity of AmyL and AmyS were highest when the concentration of xylose was $4.0 \%$. This indicates that there is an optimal expression level of prsA for heterologous protein secretion in B. subtilis, as stated in the research that there was a linear correlation between the number of cellular PrsA molecules and the number of secreted AmyQ molecules over a wide range of $p r s A$ and amyQ expression levels [31]. In addition, the single overexpression of $S R P$, ftsy, secA, secYEG, secDF, groESL operon, partial dnaK operon, sipT and sipS moderately or marginally improved 

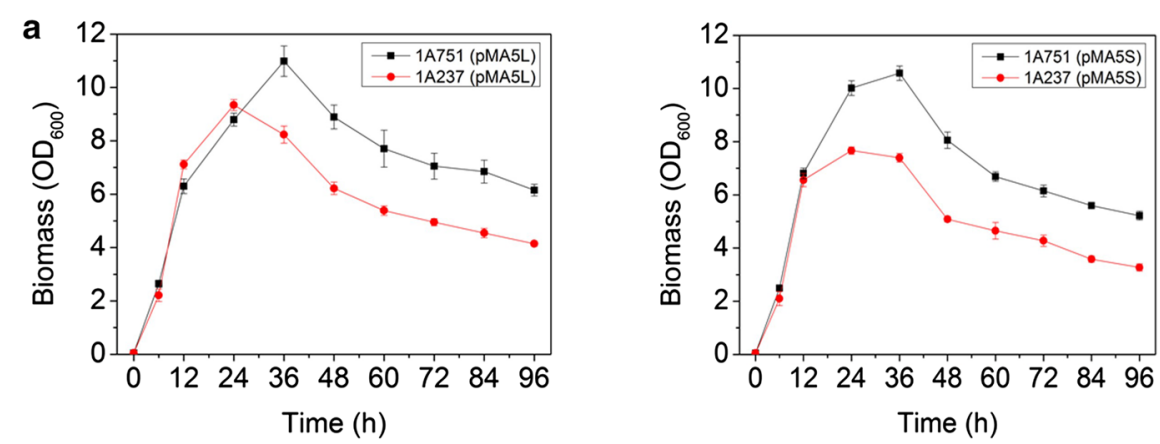

b
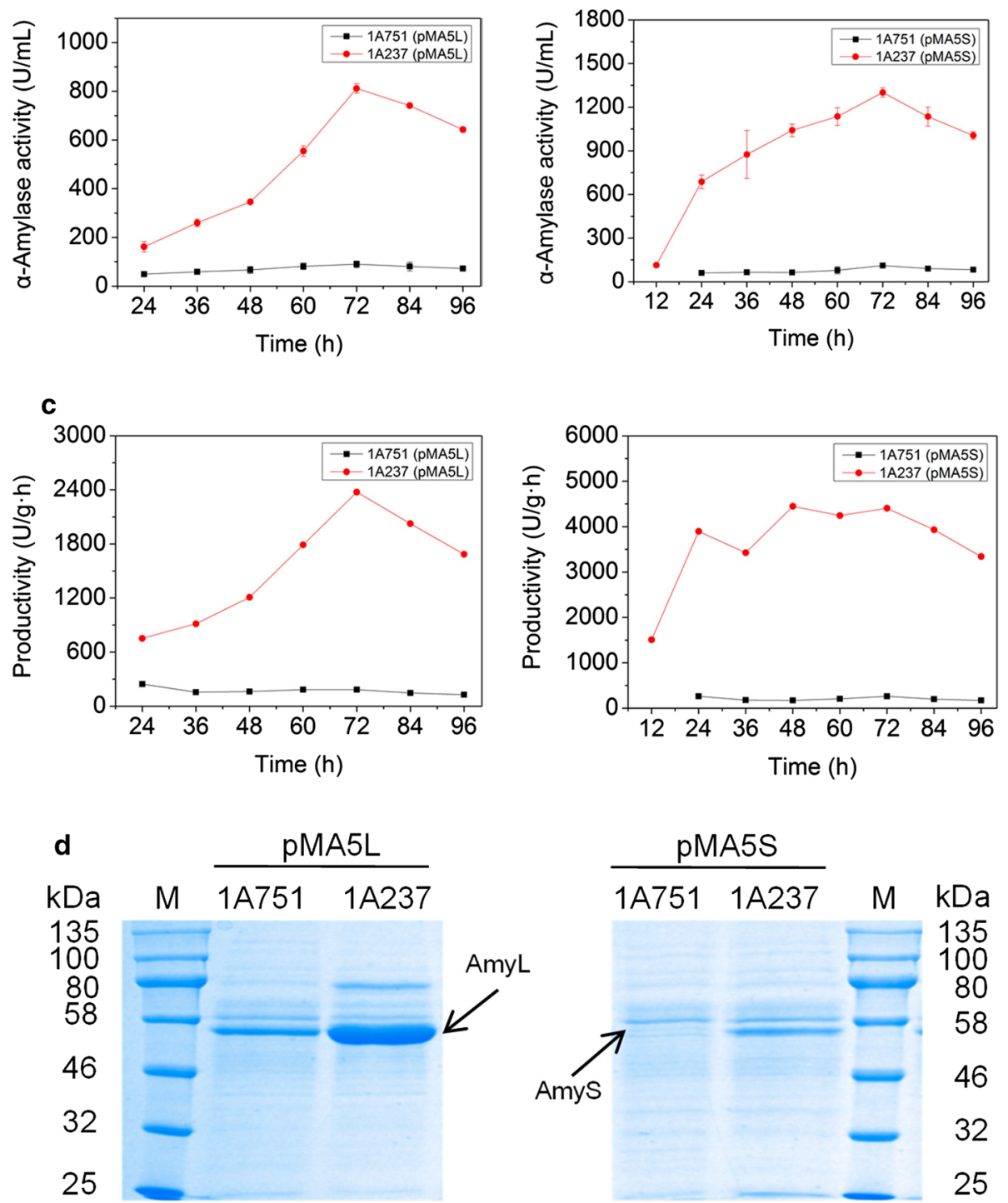

Figure 5 Characterization of engineered and parental strains. B. subtilis 1A751 (pMA5L) (parental strain), 1A237 (pMA5L) (engineered strain), 1A751 (pMA5S) (parental strain) and 1A237 (pMA5S) (engineered strain) were cultivated for $96 \mathrm{~h}$ at $37^{\circ} \mathrm{C}$ in $30 \mathrm{~mL} \mathrm{SR} \mathrm{medium} \mathrm{supplemented} \mathrm{with} 4 \%$ xylose ( $w / v)$. a Growth of the strains. b Analysis of $\alpha$-amylase (AmyL and AmyS) activity. c Analysis of productivity. d SDS-PAGE analysis of $\alpha$-amylase (AmyL and AmyS) at $72 \mathrm{~h}$. 


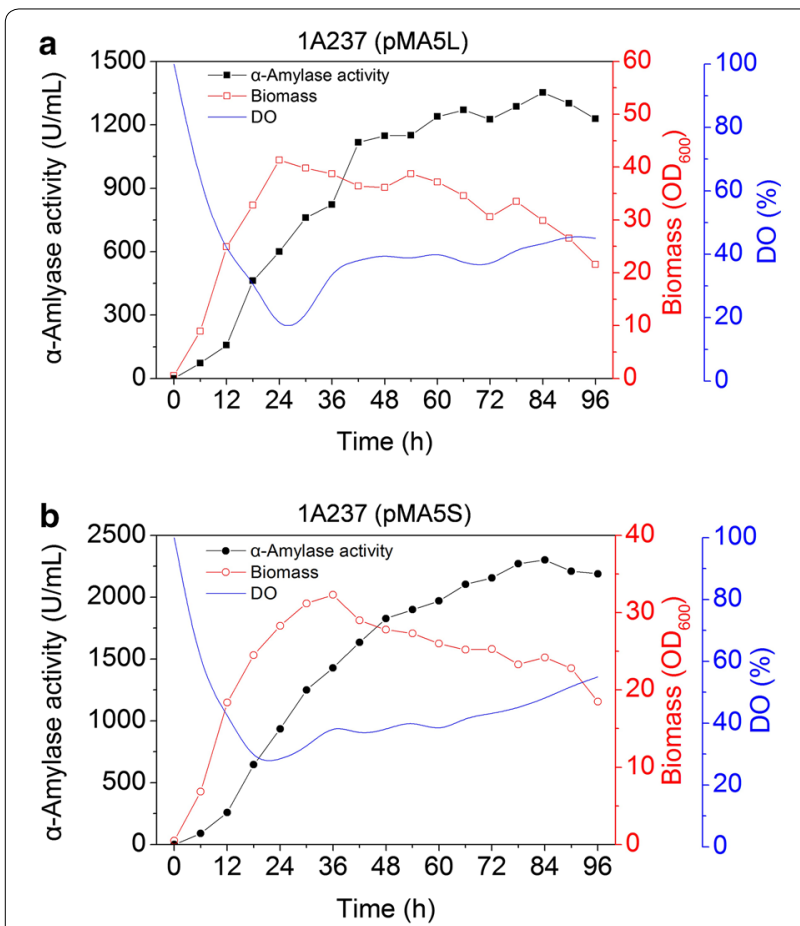

Figure 6 Production of $\alpha$-amylase in recombinant strain 1A237 by fed-batch fermentation in $7.5 \mathrm{~L}$ fermentor. a AmyL production. Solid square $\alpha$-amylase activity in medium. Hollow square biomass. Blue line DO concentration. b AmyS production. Solid circle $\alpha$-amylase activity in medium. Hollow circle biomass. Blue line DO concentration.

the production of AmyL or AmyS. However, the contribution of genes to AmyL and AmyS secretion were not completely consistent. For example, secYEG overexpression improved the production of AmyL but not AmyS; groESL operon and sipT overexpression enhanced the secretion of AmyS, but reduced AmyL production. From these results, we can know that partial components of Sec pathway have different impacts on different proteins secretion.

Based on the optimal expression level of $\operatorname{prs} A$, we overexpressed 9 screened genes or gene operons combined with prs $A$ in 1A751, respectively. The overexpression of prs $A$ and partial $d n a K$ operon resulted in the highest $\alpha$-amylase activity of both AmyL and AmyS. Dnak series are intracellular molecular chaperones mediating protein folding, minimizing aggregation and maintaining pre-proteins in translocation-competent conformations. With the overexpression of partial dnaK operon, the pre-proteins obtained stronger resistance against intracellular proteases, resulting in further improvement of heterologous protein secretion. Unexpectedly, the influence of the double-overexpression strains was not always consistent with the performance of the respective single-overexpression strain. For example, we predicted that overexpression of prsA and $\sec D F$ would contribute the most to AmyL production compared with other gene combinations; however, the $\alpha$-amylase activity of $1 \mathrm{~A} 237$ (prsA partial $d n a K$ operon) was the highest and exceeded that of $1 \mathrm{~A} 235$ (prsA secDF) by $22 \%$. In addition, the $\alpha$-amylase activity of AmyL in medium of 1A231 (prsA SRP), 1A233 (prsA secA), 1A234 (prsA secYEG), 1A236 (prsA groESL opeon) and 1A239 (prsA sipS) were even markedly lower than that of 1A23 (prsA). The similar phenomenon also appeared when AmyS was produced. Although the $\alpha$-amylase activity of AmyS in medium of 1A237 (prsA partial dnaK operon) was the highest as predicted, the $\alpha$-amylase activity of AmyS in medium of $1 \mathrm{~A} 235$ ( prsA secDF) was almost the same with that of 1A23 (prsA). Double-overexpression of prsA-secA, prsAgroESL operon, prsA-ftsy, prsA-SRP and prsA-sipS even reduced the production of AmyS compared with single overexpression of prsA. Particularly, with the overexpression of SRP operon, the secretion of AmyL and AmyS was severely impaired, which is unexpected. We suspect that excessive SRP may cause some unknown harmful effect on physiological characteristic of B. subtilis, resulting into remarkably decrease of $\alpha$-amylase production. In short, the overexpression of prs $A$ combined with some genes or gene operons which have positive effect on $\alpha$-amylase secretion reduced the production of AmyL or AmyS in some extent. These observations suggest that the interactions between components of Sec pathway have different effects on protein secretion.

By comparing the characterization of engineered and parental strains, we can see simultaneous overexpression of prs $A$ and partial dnaK operon improved the final $\alpha$-amylase activity of AmyL and AmyS by about ninefold and 12-fold and the productivity of AmyL and AmyS by approximately 13- and 17-fold, respectively. For heterologous protein secretion, a key observation is that proteins must fold rapidly if they are to avoid blocking the translocase itself or cell-wall growth sites: slowly folding proteins expose protease-sensitive sites that are not exposed in the fully folded protein. This is best illustrated by the kinetics of secretion of AmyL [36]. Pulse-chase experiments show that during secretion from $B$. licheniformis almost $100 \%$ of the synthesized protein is recovered from the culture medium. By contrast, when transferred to $B$. subtilis, $\sim 75 \%$ of the initially formed protein is degraded, and only $25 \%$ is recovered from the growth medium. The intracellular molecular chaperones (mainly DnaK series) may potentially assist AmyL and amyS to adopt loosely folded conformations that are compatible with the secretion apparatus and/or to attain configurations that are less susceptible to intracellular protease degradation. After membrane translocation, the secreted AmyL or AmyS is presumably refolded. The rate of protein 
refolding has been shown to affect the final production yield of a secretory protein [37]. Usually, a higher production yield can be attained for secretory proteins with a higher refolding rate. Increasing the production of PrsA can quicken the rate of protein refolding and improve the recovery of limited number of proteins that are substrates for this chaperone, presumably by reducing their susceptibility to extracellular proteolysis. In a word, With the combinational overexpression of both intracellular and extracytoplasmic molecular chaperones, the pre-protein and mature protein both have stronger resistance against proteases, resulting in high yields of heterologous protein in B. subtilis.

\section{Conclusion}

We have shown that the combinational overexpression of prs $A$ and partial dnaK operon significantly improved the production of AmyL and AmyS, suggesting that the stability of protein is vital for protein secretion and the deficiency of intracellular and extracellular chaperones may be main bottleneck of heterologous protein production in B. subtilis. It can thus be seen that balanced expression of Sec pathway components is crucial for efficient translocation and secretion. Moreover, we need to find out in detail how components of Sec pathway interact with each other, so that the interactions can be optimized specifically to improve the secretion of heterologous protein in B. subtilis.

\section{Methods}

\section{Bacterial strains, plasmids and growth conditions}

Bacterial strains and plasmids used in this study are listed in Tables 1 and 2, respectively. The bacterial strain Bacillus licheniformis CICC 10181 and Geobacillus stearothermophilus ATCC 31195 were used as the source of the AmyL (amyl) gene and AmyS (amys) gene, respectively. Escherichia coli DH5 $\alpha$ served as a host for cloning and plasmid preparation. Bacillus subtilis 1A751, which is deficient in two extracellular proteases (nprE, aprE), was used as a host for expression of AmyL and AmyS. The plasmid pMA5 is an E. coli/B. subtilis shuttle vector and used to clone and express AmyL and AmyS. The plasmid $\mathrm{pDD}$ is an integration vector which is derivative from $\mathrm{pDL}$ and just contains the front and back parts of $a m y E$. Transformants of $E$. coli and B. subtilis were selected on Luria-Bertani (LB) agar [1\% (w/v) Trytone, 0.5\% (w/v) Yeast extract, $1 \%(\mathrm{w} / \mathrm{v}) \mathrm{NaCl}$ and $2 \%(\mathrm{w} / \mathrm{v})$ agar], supplemented with ampicillin $(100 \mu \mathrm{g} / \mathrm{mL})$ or kanamycin $(50 \mu \mathrm{g} / \mathrm{mL})$ depending on the plasmid antibiotic marker. Unless otherwise specified, integrated B. subtilis mutants were selected on LB agar, containing kanamycin $(50 \mu \mathrm{g} /$ $\mathrm{mL}$ ) or chloramphenicol $(12.5 \mu \mathrm{g} / \mathrm{mL})$. E. coli DH5 $\alpha$ was incubated in LB medium supplemented with ampicillin
$(100 \mu \mathrm{g} / \mathrm{mL})$ at $37^{\circ} \mathrm{C}$. B. subtilis was cultivated in SR medium $[1.5 \%(\mathrm{w} / \mathrm{v})$ tryptone, $2.5 \%(\mathrm{w} / \mathrm{v})$ yeast extract and $0.3 \%(\mathrm{w} / \mathrm{v}) \mathrm{K}_{2} \mathrm{HPO}_{4}, \mathrm{pH}$ 7.2] contained additionally kanamycin $(50 \mu \mathrm{g} / \mathrm{mL})$ or chloramphenicol $(12.5 \mu \mathrm{g} / \mathrm{mL})$ at $37^{\circ} \mathrm{C}$. All of the strains were incubated under a shaking condition at $200 \mathrm{rpm}$. All of the experiments were repeated at least three times and mean values were used for comparison.

\section{Primers and oligonucleotides}

Polymerase chain reaction (PCR) primers and oligonucleotides used in this study were synthesized by GENEWIZ (Suzhou, China) and listed in Additional file 2: Table S1.

\section{General manipulation}

Standard molecular techniques including E. coli transformation were carried out according to Sambrook et al. [38]. B. subtilis was naturally transformed using "Paris Method" [39, 40]. PCRs were performed using PrimeSTAR Max DNA Polymerase (TaKaRa, Japan). DNA fragments and PCR products were excised from a $0.8 \%$ agarose gel and purified by E.Z.N.A. ${ }^{m}$ Gel Extraction Kit (200) (Omega Bio-tek, Inc., USA) according to the manufactures' instruction. E.Z.N.A. ${ }^{\mathrm{m}}$ Plasmid Mini Kit I (Omega Bio-tek, Inc., USA) was applied for plasmid extraction according to the manufactures' instruction. Genomic DNA isolation was carried out by TIANamp Bacteria DNA Kit (TIANGEN BIOTECH (BEIJING) CO., LTD., China). All the DNA constructs were sequenced by GENEWIZ (Suzhou, China).

\section{Construction and transformation of the plasmid for secreted AmyL or AmyS}

Based on the nucleotide sequence of the gene encoding AmyL or AmyS, the primer pairs amyL-F/amyL-R or amyS-F/amyS-R (Additional file 2: Table S1) were designed to amplify the gene amyl or amys from Bacillus licheniformis CICC 10181 or Geobacillus stearothermophilus ATCC 31195 with the introduction of EcoRI and XhoI restriction site at $5^{\prime}$ of the forward and reverse primers, respectively. The gene amyl or amys contains their native signal peptide $\mathrm{SP}_{a m y l}$ or $\mathrm{SP}_{a m y s}$. The EcoRI$X h o I$ digested fragments amyl and amys were ligated with pET28-a $(+)$ linearized by the same enzymes, resulting in pET28L and pET28S under the control of the T7-promoter and T7-terminator, respectively.

The DNA fragments AmyL gene-T7 terminator (amylT7) and AmyS gene-T7 terminator (amys-T7) were amplified from pET28L and pET28S plasmids as template with the primer pairs amyLT-F/R and amyST-F/R, which contained NdeI and BamHI restriction site at $5^{\prime}$ of the forward and reverse primers, respectively (Additional file 1: Figure S1). It was followed by ligation of the 
NdeI-BamHI digested amyl-T7 or amys-T7 fragment with pMA5 linearized by the same enzymes, which is an E. coli/B. subtilis shuttle vector containing an widely used strong promoter $\mathrm{P}_{\text {HpaII }}$ and a strong $B$. subtilis ribosome binding site (RBS), resulting in the recombinant plasmid pMA5L or pMA5S. B. subtilis 1A751 were transformed according to the method as previously described with the control plasmid pMA5 and expression plasmids pMA5L and pMA5S, respectively.

\section{Construction of the integration vectors for genes overexpression}

All the integration vectors (Table 2) used in this study were constructed by a sequence-independent "simple cloning" method without the need for restriction and ligation enzymes [41]. To develop an integration-vector system for co-overexpression of two different genes, we constructed an integration plasmid pDDXG in advance. First of all, a 1.4-kb insertion fragment (the promoter $\mathrm{P}_{x y l A}$ ) was subcloned into an 8.1-kb pDD vector backbone, yielding a 9.5-kb plasmid, pDDX. The linear vector backbone was amplified by using the forward primer pDD-F (5'GTTCACTTAAA TCAAAGGGGGAAATAG AAGTCTCGTTCCGACAGTTGGCA3') and the reverse primer pDD-R (5'CGTTTTACAACGTCGTGA CTGGGAAACCGGGAAT TCTCAT GTTTGACAG $\left.\underline{\mathrm{C}} 3^{\prime}\right)$. pDD-F/R contain the last $25 \mathrm{bp}$ of the $3^{\prime}$ terminus of the insertion sequence (underlined) and the first $25 \mathrm{bp}$ of the $5^{\prime}$ terminus of the vector sequence (bold). Similarly, the insertion fragment was amplified by primers xylA-F (5'AAG CTGTCAAACATGAGAATTCCCG GTTTCCCAGTCACGACGT TGTAAAAC $\left.3^{\prime}\right)$ and xylAR (5'TGCCAACTGTCGGAACGAGACTTCTATTT CC CCCTTTGATTTAAGTGAAC $3^{\prime}$ ). xylA-F/R have the reverse complementary sequences of $\mathrm{pDD}-\mathrm{F} / \mathrm{R}$, respectively. Then, the DNA multimer is generated based on these DNA templates by prolonged overlap extension PCR (POE-PCR). Eventually, the POE-PCR products (DNA multimer) were transformed into competent $E$. coli DH5 $\alpha$ directly, yielding the recombinant plasmid pDDX. Using primers grac-F/R and $\mathrm{pDDX}-\mathrm{F} / \mathrm{R}$, the $\mathrm{P}_{\text {grac }}$ fragment and linear vector backbone were amplified from pHT43 and pDDX, respectively. Subsequently, the integration vector $\mathrm{pDDXG}$ was constructed as described above. In the same way, pDDXBG and pDDXGB into which the gene bgaB was inserted under the control of $\mathrm{P}_{x y l A}$ and $\mathrm{P}_{\text {grac }}$, respectively, were also constructed.

All the integration vectors for overexpression of the corresponding single gene or gene operon involved in Sec pathway in B. subtilis were constructed based on the plasmid pDDXG. 19 genes (ffh, hbs, scr, ftsy, csaA, secA, secY, secE, secG, secDF, yrbF, spoIIIJ, yqjG, sipT, sipS, sipU, sipV, sipW and prsA) and two gene operons (groESL operon and partial $d n a K$ operon) were amplified using corresponding primers (Additional file 2: Table S1) and B. subtilis 168 genomic DNA as the template. Meanwhile, two artificial gene operons (SRP operon and secYEG operon) were assembled. The genes ffh, hbs (containing its own ribosome-binding site) and scr were amplified using primers ffh-F2/R2, hbs-F2/R2 and scr-F2/R2, respectively, and then the $S R P$ operon was assembled by POE-PCR using primers ffh-F2/scr-R2. The genes secY, $\sec E$ (containing its own ribosome-binding site) and secG (containing its own ribosome-binding site) were amplified using primers secY-F2/R2, secE-F2/R2 and secG-F2/ $\mathrm{R} 2$, respectively, and the secYEG operon was assembled by POE-PCR using primers secY-F2/secG-R2. The DNA fragments of 19 genes and 4 gene operons above were then inserted into the plasmid pDDXG, successively, under the control of the promoter $\mathrm{P}_{x y l A}$, resulting into 23 corresponding integration vectors (Table 2 ).

The five genes ftsy, secA, secDF, sipT and sipS and four operons SRP operon, secYEG operon, groESL operon and partial $d n a K$ operon were amplified by PCR from genomic DNA of B. subtilis 168 or corresponding plasmids using primers (Additional file 2: Table S1). The vector backbone was amplified from pDD23 with primers pDD23-F/R. By the "simple cloning" method as described above, nine corresponding integration plasmids were constructed successfully.

\section{Isolation of total RNA and Real-time PCR}

The culture was harvested at $48 \mathrm{~h}$. Total RNA of bacteria was isolated by using SV total RNA isolation kit (Cat. Z3100, Promega). The cDNA chain was synthesized by using Reverse Transcription System (Cat. A3500, Promega). Real-time PCR was performed by using Real time PCR Kit (Cat. DRR041 S, TaKaRa). The target genes were amplified using respective primers. B. subtilis $16 \mathrm{~s}$ rDNA was amplified as control and the PCR protocol was as follows: $2 \mathrm{~min}$ at $50^{\circ} \mathrm{C}, 10 \mathrm{~min}$ at $95^{\circ} \mathrm{C}$, and then 35 cycles consisting of $45 \mathrm{~s}$ at $95^{\circ} \mathrm{C}, 1 \mathrm{~min}$ at $52^{\circ} \mathrm{C}$, and $30 \mathrm{~s}$ at $72^{\circ} \mathrm{C}$. Reactions were carried out in real-time PCR detection system (IQ5, Bio-RAD).

\section{Fed-batch fermentation in $7.5 \mathrm{~L}$ fermentor}

The recombinant B. subtilis $1 \mathrm{~A} 751$ (pMA5L) and $1 \mathrm{~A} 751$ (pMA5S) were used to scale up fermentation in $7.5 \mathrm{~L}$ BIO FLO 310 fermentor (New Brunswick Scientific co Inc., USA), with a fed-batch strategy. The airflow rate was 6.0 L/min, and dissolved oxygen tension was maintained between 20 and $40 \%$ air saturation by automatic adjustment of speed of the stirrer. The temperature was kept at $37^{\circ} \mathrm{C}$ and the $\mathrm{pH}$ was controlled at $\mathrm{pH} 7.0$. Foam was controlled by the addition of a silicone-based anti-foaming agent. The fermentation medium (1.5\% tryptone, $2.5 \%$ 
yeast extract, $2.0 \%$ soluble starch and $0.3 \% \mathrm{~K}_{2} \mathrm{HPO}_{4}$ ) was supplemented with $50 \mu \mathrm{g}$ kanamycin $/ \mathrm{mL}$ and $100 \mu \mathrm{g}$ chloramphenicol $/ \mathrm{mL}$. The fermentation was performed with an initial working volume of $3.5 \mathrm{~L}$. When the cell growth rate was constant, the substrate fed-batch mode was started by adding $8.0 \%$ soluble starch at a constant flow rate, until the final concentration of soluble starch was up to $4.0 \%$. Cell growth was monitored by measuring $\mathrm{OD}_{600}$ of the fermentation broth. The activity of $\alpha$-amylase was determined by measuring the supernatant of broth.

\section{Enzyme assays}

The definition of $\alpha$-amylase is described below. One unit of enzyme is the amount of amylase needed to complete the hydrolysis of starch into dextrin per minute at $70^{\circ} \mathrm{C}$ and $\mathrm{pH}$ 6.0. The measurement was done according to Chinese Industrial Standard (GB 8275-2009). The calculation of the enzyme activity was based on the formula below. $X=c \times n \times 16.67$, where $X$ is the enzyme activity of the sample $(\mathrm{U} / \mathrm{mL}), c$ is the concentration of the control enzyme $(\mathrm{U} / \mathrm{mL})$ corresponded with the absorbance and $n$ is the dilution fold. $\beta$-galactosidase assay was measured as described [42].

\section{SDS-PAGE analysis}

Culture samples $(1 \mathrm{~mL})$ were harvested and the supernatant was separated from the culture medium by centrifugation $\left(12,000 \mathrm{~g}, 10 \mathrm{~min}, 4^{\circ} \mathrm{C}\right)$. After adding $5 \times$ SDS-PAGE sample buffer, the supernatants were boiled for $10 \mathrm{~min}$ and proteins were separated in SDS-PAGE using the NuPAGE 10\% Bis-Tris Gel (Novex by Life Technologies, USA) in combination with MOPS SDS Running Buffer (Invitrogen Life Technologies, USA). PageRuler Prestained Protein Ladder (Invitrogen Life Technologies, USA) was used to determine the apparent molecular weight of separated proteins. Proteins were visualized with Coomassie Brilliant Blue.

\section{Additional files}

Additional file 1: Figure S1. The construction of all the integration plasmids used in this study.

Additional file 2: Table S1. Primers used in this study.

\section{Authors' contributions}

JC, GF and DZ designed the experiments; JC performed the experiments; JC, PZ, DZ and JW wrote the manuscript; and all authors contributed to the discussion of the research. All authors read and approved the final manuscript.

\section{Author details}

${ }^{1}$ Department of Biological Engineering, School of Chemical Engineering and Technology, Tianjin University, Tianjin 300072, People's Republic of China.

${ }^{2}$ Tianjin Institute of Industrial Biotechnology, Chinese Academy of Sciences, Tianjin 300308, People's Republic of China. ${ }^{3}$ Key Laboratory of Systems Microbial Biotechnology, Chinese Academy of Sciences, Tianjin 300308, People's
Republic of China. ${ }^{4}$ National Engineering Laboratory for Industrial Enzymes, Tianjin 300308, People's Republic of China.

\section{Acknowledgements}

Authors would like to express their thanks to financial supports from State Key Development 973 Program for Basic Research of China (2013CB733601), National Nature Science Foundation of China (31370089, 31200036), and the Key Projects in the Tianjin Science \& Technology Pillar Program (12ZCZDSY12700, 14ZCZDSY00065).

\section{Compliance with ethical guidelines}

\section{Competing interests}

The authors declare that they have no competing interests.

Received: 5 March 2015 Accepted: 11 June 2015

Published online: 26 June 2015

\section{References}

1. Nijland R, Kuipers OP (2008) Optimization of protein secretion by Bacillus subtilis. Recent Pat Biotechnol 2:79-87

2. Westers L, Westers H, Quax WJ (2004) Bacillus subtilis as cell factory for pharmaceutical proteins: a biotechnological approach to optimize the host organism. Biochim Biophys Acta 1694:299-310

3. Dong H, Zhang D (2014) Current development in genetic engineering strategies of Bacillus species. Microb Cell Fact 13:63

4. Phan TT, Nguyen HD, Schumann W (2012) Development of a strong intracellular expression system for Bacillus subtilis by optimizing promoter elements. J Biotechnol 157:167-172

5. Diao L, Dong Q, Xu Z, Yang S, Zhou J, Freudl R (2012) Functional implementation of the posttranslational SecB-SecA protein-targeting pathway in Bacillus subtilis. Appl Environ Microbiol 78:651-659

6. Song Y, Nikoloff JM, Zhang D (2015) Improving protein production on the level of regulation both of expression and secretion pathways in Bacillus subtilis. J Microbiol Biotechnol. doi:10.4014/jmb.1501.01028

7. Struck JCR, Hartmann RK, Toschka HY, Erdmann VA (1989) Transcription and processing of Bacillus subtilis small cytoplasmic RNA. Mol Gen Genet 215:478-482

8. Harold T, Albert B, Jan DHJ, Sierd B, Jan M van D (2000) Signal peptidedependent protein transport in Bacillus subtilis: a genome-based survey of the secretome. Mol Biol Rev 64:515-547

9. Nakamura K, Yahagi Si, Yamazaki T, Yamane K (1999) Bacillus subtilis histone-like protein, HBsu, is an integral component of a SRP-like particle that can bind the alu domain of small cytoplasmic RNA. J Biol Chem 274:13569-13576

10. Angelini S, Deitermann S, Koch HG (2005) Fts Y, the bacterial signal-recognition particle receptor, interacts functionally and physically with the SecYEG translocon. EMBO Rep 6:476-481

11. Müller JP, Bron S, Venema G, Dij JMv (2000) Chaperone-like activities of the CsaA protein of Bacillus subtilis. Microbiology 146:77-88

12. Leloup L, Driessen AJM, Freudl R (1999) Differential dependence of levansucrase and $\alpha$-amylase secretion on SecA (Div) during the exponential phase of growth of Bacillus subtilis. J Bacteriol 181:1820-1826

13. Kakeshita H, Kageyama Y, Ara K, Ozaki K, Nakamura K (2010) Enhanced extracellular production of heterologous proteins in Bacillus subtilis by deleting the C-terminal region of the SecA secretory machinery. Mol Biotechnol 46:250-257

14. Mulder KC, Bandola J, Schumann W (2013) Construction of an artificial secYEG operon allowing high level secretion of alpha-amylase. Protein Expr Purif 89:92-96

15. Kedrov A, Kusters I, Krasnikov VV, Driessen AJ (2011) A single copy of SecYEG is sufficient for preprotein translocation. EMBO J 30:4387-4397

16. Lycklama a Nijeholt JA, de Keyzer J, Prabudiansyah I, Driessen AJ (2013) Characterization of the supporting role of SecE in protein translocation. FEBS Lett 587:3083-3088

17. Bolhuis A, Broekhuizen CP, Sorokin A, van Roosmalen ML, Venema G, Bron S et al (1998) SecDF of Bacillus subtilis, a molecular siamese twin required for the efficient secretion of proteins. J Biol Chem 273:21217-21224 
18. Ling Lin F, Zi Rong X, Wei Fen L, Jiang Bing S, Ping L, Chun Xia H (2007) Protein secretion pathways in Bacillus subtilis: implication for optimization of heterologous protein secretion. Biotechnol Adv 25:1-12

19. Saller MJ, Otto A, Berrelkamp-Lahpor GA, Becher D, Hecker M, Driessen AJ (2011) Bacillus subtilis YqjG is required for genetic competence development. Proteomics 11:270-282

20. Schallmey M, Singh A, Ward OP (2004) Developments in the use of Bacillus species for industrial production. Can J Microbiol 50:1-17

21. Li W, Zhou X, Lu P (2004) Bottlenecks in the expression and secretion of heterologous proteins in Bacillus subtilis. Res Microbiol 155:605-610

22. Bos JW, Frenken LG, Verrips CT, Visser C (1998) US5804409

23. Quax WJ, Kerkman R, Broekhuizen CP (2002) US6410262

24. Nouaille S, Morello E, Cortez-Peres N, Le Loir Y, Commissaire J, Gratadoux $J J$ et al (2006) Complementation of the Lactococcus lactis secretion machinery with Bacillus subtilis SecDF improves secretion of staphylococcal nuclease. Appl Environ Microbiol 72:2272-2279

25. Malten M, Nahrstedt H, Meinhardt F, Jahn D (2005) Coexpression of the type I signal peptidase gene sipM increases recombinant protein production and export in Bacillus megaterium MS941. Biotechnol Bioeng 91:616-621

26. Wu SC, Ye R, Wu XC, Ng SC, Wong SL (1998) Enhanced secretory production of a single-chain antibody fragment from Bacillus subtilis by coproduction of molecular chaperones. J Bacteriol 180:2830-2835

27. Lindholm A, Ellmen U, Tolonen-Martikainen M, Palva A (2006) Heterologous protein secretion in Lactococcus lactis is enhanced by the Bacillus subtilis chaperone-like protein PrsA. Appl Microbiol Biotechnol 73:904-914

28. Kakeshita H, Kageyama Y, Endo K, Tohata M, Ara K, Ozaki K et al (2011) Secretion of biologically-active human interferon-beta by Bacillus subtilis. Biotechnol Lett 33:1847-1852

29. Kontinen VP, Saris P, Sarvas M (1991) A gene (prsA) of Bacillus subtilis involved in a novel, late stage of protein export. Mol Microbiol $5: 1273-1283$

30. Kontinen VP, Sarvas M (1993) The PrsA lipoprotein is essential for protein secretion in Bacillus subtilis and sets a limit for high-level secretion. Mol Microbiol 8:727-737

31. Vitikainen M, Pummi T, Airaksinen $U$, Wahlstrom $E, W u H$, Sarvas M et al (2001) Quantitation of the capacity of the secretion apparatus and requirement for PrsA in growth and secretion of alpha-amylase in Bacillus subtilis. J Bacteriol 183:1881-1890
32. Liu YH, Lu FP, Li Y, Yin XB, Wang Y, Gao C (2008) Characterisation of mutagenised acid-resistant alpha-amylase expressed in Bacillus subtilis WB600. Appl Microbiol Biotechnol 78:85-94

33. Niu D, Zuo Z, Shi GY, Wang ZX (2009) High yield recombinant thermostable alpha-amylase production using an improved Bacillus licheniformis system. Microb Cell Fact 8:58

34. Vitikainen M, Hyyrylainen HL, Kivimaki A, Kontinen VP, Sarvas M (2005) Secretion of heterologous proteins in Bacillus subtilis can be improved by engineering cell components affecting post-translocational protein folding and degradation. J Appl Microbiol 99:363-375

35. Chen J, Gai Y, Fu G, Zhou W, Zhang D, Wen J (2015) Enhanced extracellular production of alpha-amylase in Bacillus subtilis by optimization of regulatory elements and over-expression of PrsA lipoprotein. Biotechnol Lett 37:899-906

36. Jensen CL, Harwood C, Jørgensen ST, Stephenson K (2000) Cell-associated degradation affects the yield of secreted engineered and heterologous proteins in the Bacillus subtilis expression system. Microbiology 146:2583-2594

37. Stephenson K, Carter NM, Harwood CR, Petit-Glatron MF, Chambert R (1998) The influence of protein folding on late stages of the secretion of alpha-amylases from Bacillus subtilis. FEBS Lett 430:385-389

38. Green MR, Sambrook J (2012) Molecular cloning: a laboratory manual, 4th edn. Cold Spring Harbor Laboratory Press, New York

39. Harwood CR, Cutting SM (1990) Molecular biological methods for Bacillus. Wiley, Chichester

40. Spizizen J (1958) Transformation of biochemically deficient strains of Bacillus subtilis by deoxyribonucleate. Proc Natl Acad Sci USA 44:1072-1078

41. You C, Zhang XZ, Zhang YH (2012) Simple cloning via direct transformation of PCR product (DNA Multimer) to Escherichia coli and Bacillus subtilis. Appl Environ Microbiol 78:1593-1595

42. Daniel R. Zeigler PD (2002) Bacillus genetic stock center catalog of strains, 7th edn. Baillus Genetic Stock Center, Columbus, pp 4

\section{Submit your next manuscript to BioMed Central and take full advantage of:}

- Convenient online submission

- Thorough peer review

- No space constraints or color figure charges

- Immediate publication on acceptance

- Inclusion in PubMed, CAS, Scopus and Google Scholar

- Research which is freely available for redistribution

Submit your manuscript at 